Lim et al., Afr J Tradit Complement Altern Med., (2017) 14 (5): 113-120

https://doi.org/10.21010/ajtcam.v14i5.15

\title{
EFFECTS OF GLYCYRRHIZIN PRE-TREATMENT ON TRANSIENT ISCHEMIC BRAIN INJURY IN MICE
}

\section{Chiyeon Lim ${ }^{1 \dagger}$, Sehyun Lim ${ }^{2 \dagger}$, Young-Jun Lee ${ }^{3}$, Bokcheul Kong ${ }^{4}$, Byoungho Lee ${ }^{5}$, Chang- Hyun Kim ${ }^{1}$, Buyeo Kim ${ }^{6}$, Suin $\mathrm{Cho}^{7^{*}}$}

${ }^{1}$ College of Medicine, Dongguk University, Ilsandong-gu, Gyeonggi-do, 10326, Republic of Korea. ${ }^{2}$ School of Public Health, Far East University, Chungbuk,27601, Republic of Korea. ${ }^{3}$ Lee Young Jun Clinic of Korean Medicine, Cheonan-si, Chungcheongnam-do,331-963, Republic of Korea. ${ }^{4}$ Samse Oriental Medical Hospital, Gumjeong-gu, Busan-si, 46302, Republic of Korea. ${ }^{5}$ Hongjae Korean Medicine Clinic, Youngdeungpo-gu, Seoul-si, 150051, Republic of Korea. ${ }^{6}$ Department of Medical Research, Korea Institute of Oriental Medicine, Daejeon,34054, Republic of Korea. ${ }^{7}$ School of Korean Medicine, Yangsan Campus of Pusan National University, Yangsansi, 50612, Republic of Korea. ${ }^{\dagger}$ Contributed equally to this work

*Corresponding Author E-mail:sicho@pusan.ac.kr

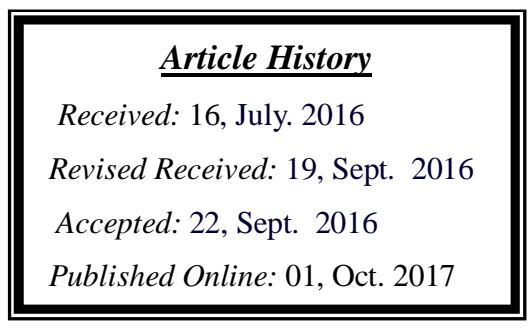

\begin{abstract}
Background: Ischemia-induced brain damage is the leading cause of adult disability and the fifth leading cause of death, and thus, the development of anti-apoptotic neuro-protective therapeutic agents is viewed as an attractive developmental strategy. Glycyrrhizin is the main sweet component in licorice and has a number of pharmacological activities, which include neuro-protective, anti-fungal, and anti-cariogenic activities. This study was undertaken to investigate the effects of glycyrrhizin on ischemia-induced brain damage.

Materials and Methods: In infarct volumes and the levels of several apoptosis-related proteins, caspase-3, $8,9, \mathrm{Bcl}-\mathrm{xL}, \mathrm{Bcl}-2$, and their activities in the brains of middle cerebral artery occlusion (MCAO) treated mice were measured using western blotting methods.

Results: Single pre-treatment with glycyrrhizin $(10-100 \mathrm{mg} / \mathrm{kg})$ at 2 hours before MCAO significantly reduced infarct volumes at $24 \mathrm{~h}$ after MCAO. In addition, glycyrrhizin effectively inhibited the activations of caspase- 3 and -9 and the down-regulation of Bcl-xLprotein caused by MCAO.

Conclusion: The neuro-protective effect of glycyrrhizin was found to be due to its regulation of apoptosisrelated proteins signals. The authors suggest glycyrrhizin be considered a potential candidate for the treatment of ischemia induced brain damage.
\end{abstract}

Keywords: Glycyrrhizin, licorice, stroke, apoptosis

Abbreviations: MCAO, middle cerebral artery occlusion; rCBF, relative cerebral blood flow; TTC, 2,3,5Triphenyl-tetrazolium chloride. 


\section{Introduction}

Glycyrrhizin (glycyrrhizic acid) is one of main constituents of Glycyrrhiza glabra or $G$. uralensis (licorice) roots, and has been shown to have a number of pharmacological activities, which including neuro-protective(Kim et al., 2011; 2012; Luo et al., 2013), anti-fungal(Guo, 1991), and anticariogeniceffects (Segal et al., 1985). Isoliquiritigenin, a natural phenol isolated from licorice, has been reported to have protective effects on transient MCAO induced focal cerebral ischemia in rats(Zhan and Yang, 2006). In total, 122 compounds have been isolated from G. uralensis and extensively tested using various bioassay methods(Ji et al., 2016; Ota et al., 2015; Yang et al., 2015b; Zhu et al., 2016).Kumagai et al.(1957) reported glycyrrhizincompetitively inhibits the metabolism of corticosteroidsand that it has a corticosteroid-like effect (Kumagai et al., 1957).

Corticosteroidsare often administered by clinicians, butevidence concerning their effects on acute cerebral infarction is lacking. However, corticosteroids are believed to reduce cytotoxic and vasogenic brain edema when administered to ischemic cerebral stroke (Faraji et al., 2009; Feigin et al., 2005; Sandercock and Soane, 2011).

Stroke is a leading cause of adult death and permanent disability, and ischemic strokeaccounts for around $80 \%$ of strokes (Poisson et al., 2014).Furthermore, the incidence of stroke is increasing among young adults(Bejot et al., 2016).

Ischemia/reperfusion-induced stroke can be characterized by focal loss of brain blood circulation, and often leads to neuronal injury or death (Hadadha et al., 2015; Zhao et al., 2015).Kim et al.(2012) concluded that the anti-inflammatory and anti-oxidative effects of glycyrrhizin were responsible for its neuroprotective effect post-ischemia in a rat MCAO model (Kim et al., 2012). However, the effects of glycyrrhizinhave not been determined in a mouse MCAO model of ischemia. Thus, in the present study, we sought to determine whether glycyrrhizinprotects against ischemia/reperfusion induced brain damage and to identify the mechanism responsible in a mouse MCAO model.

\section{Materials and Methods Glycyrrhizin treatment}

Glycyrrhizin was purchased from Sigma-Aldrich (Millipore Sigma, MO, USA), dissolved in dimethyl sulfoxide (DMSO) to concentration of $100 \mathrm{mg} / \mathrm{ml}$, and then again diluted with tap water to concentration of $2 \mathrm{mg} / \mathrm{ml}$.

\section{Animal model}

The animal experiment protocol was approved by the ethics committee of our institution (approval number, PNU-2016-1087). Male SPF C57BL/6 mice (DaehanBiolink, Chungbuk, Korea) (22-25 g) were housed in a humidity and temperature controlled environment under a $12 \mathrm{~h}$ light cycle, and provided food and water ad libitum. For the experiment animals were randomly divided into five groups, namely, the sham control group (members of which underwent surgery but not MCAO), the non-glycyrrhizin treated MCAO group (the MCAO control group), and the 10,30 , or $100 \mathrm{mg} / \mathrm{kg}$ glycyrrhizin treated groups (the 10,30 , and $100 \mathrm{mg} / \mathrm{kg}$ groups)each group consisted of at least 15 animals.

Before MCAO, mice in glycyrrhizin treatment groups were orally administered 10, 30, or 100 $\mathrm{mg} / \mathrm{kg}$ of body weight $1 \mathrm{hr}$ before commencing the MCAO procedure (Fig. 1(A)). MCAO was conducted under $2 \%$ isoflurane anesthesia. During surgery, rectal temperature was maintained at $37 \pm 0.5^{\circ} \mathrm{C}$ with a heating pad, andrelative cerebral blood flow (rCBF) was monitored using a laser Doppler blood flow system (moorVMS-LDF, Moor Instruments, Devon, UK). Briefly, under a stereo microscope (Nikon745, Tokyo), the left external carotid artery and the left common carotid artery were bound with 4/0 silk sutures (Ethicon Inc., NJ, USA).Theleft middle cerebral artery was then occluded using a filament $(11 \mathrm{~mm}$ length of $8 / 0$ nylon suture with a silicon-coated tip), and after $2 \mathrm{~h}$ of occlusion, the filament was withdrawn. The success of the procedure was confirmed by a reduction in $\mathrm{rCBF}$ of $>80 \%$ during the $2 \mathrm{~h}$ ischemic period, and a sharp increase in $\mathrm{rCBF}$ to $>90 \%$ of baseline during reperfusion. Surgical procedures performed in an identical 
manner in the sham group but the left middle cerebral artery was not occluded.Mice were anesthetized and sacrificed24 hrafter commencing MCAO.

(A)

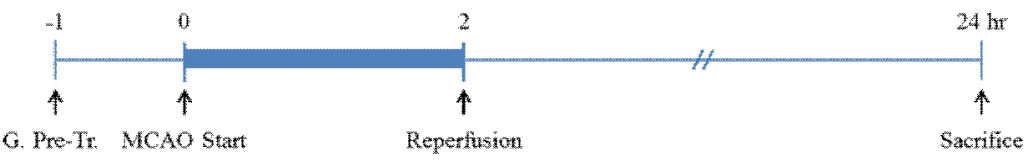

(B)


Figure 1: Design of the MCAO model and representative group images. (A) Glycyrrhizin was pre-treated 1 $\mathrm{hr}$ before commencing MCAO, and mice were sacrificed $24 \mathrm{hr}$ after commencing MCAO (24h post$\mathrm{MCAO}$ ). Harvested brain slices were kept in deep freezer for protein assays, or stained with TTCto measure infarct volumes. (B) Ischemic regions were identified as pale regions in coronal slices.

\section{Measurement of infarct volumes}

Brains harvested at $24 \mathrm{~h}$ post-MCAO were immediately sectioned at $1 \mathrm{~mm}$ coronal sections to obtain 10 sections/brain. Sections were then incubated with 2\% 2,3,5-triphenyl-tetrazolium chloride (TTC) solution for $17 \mathrm{~min}$ at $25^{\circ} \mathrm{C}$ and fixed in $10 \%$ neutral buffered formalin for more than $2 \mathrm{hr}$. Digital images were obtained using a digital camera, and infarct and relative edema volumes were calculated using Image $\mathbf{J}$ software (NIH, Maryland, USA).

\section{Neurological deficit scores}

Neurological deficit scores were assessed at 24 hrpost-MCAOusing a five-point scale. Briefly, the scores were calculated as follows: 0 , no neurological deficit; 1 , failure to extend right forepawand a reduced grip; 2, spontaneous movement in all directions and circling to the rightwhen the tail was pulled; 3 , circling or walking to the rightwhen stimulated; 4 , unresponsive to stimulation or stroke-related death.

\section{Western blot analysis}

Ipsi-lateral brain tissues including hippocampus and cortex were extracted and homogenized in tissue lysis buffer. Proteins were separated by $10 \%$ sodium dodecyl sulfate-polyacrylamide gel electrophoresis (SDS-PAGE), and then transferred to nitrocellulose membranes (Whatman, Maidstone, UK).Membranes were blocked using 5\% skim milk in TBST buffer for $1 \mathrm{hr}$ at room temperature and then incubated overnight at $4^{\circ} \mathrm{C}$ with specific antibodies for caspase-3, -8, 9, Bcl-xL, Bcl-2 (1:1000), and $\beta$-actin (1:2000) for internal control. Antibodies listed above were purchased from Cell Signaling Technology (Danvers, MA, USA). After overnight incubation, horse radish peroxidase (HRP) conjugated goat anti-rabbit IgG, pAb (1:5000) and HRP conjugated goat anti-mouse $\operatorname{IgG}$ pAb (1:3000) were added for 2 hours. Membranes were then treated with ECL solution (GenDEPOT, Houston, TX, USA) and protein bands were detected using a photosensitive luminescent analyzer system (Amersham ${ }^{\mathrm{TM}}$ Imager 600, UK). Relative 
protein amounts were analyzedusing the Image J program (NIH, Maryland, USA) versus $\beta$-actin.

\section{Statistical analysis}

One-way ANOVA was used to determine the statistical significance of differences. Data are expressed as means \pm standard deviations (means \pm SD). SIGMAPLOT 12.0 version was used to statistical analysis, and $p$-values of $\leq 0.05$ were considered statistically significant.

\section{Results \\ Effects of glycyrrhizin on behavioral deficits and infarct volumes}

At $24 \mathrm{~h}$ post-MCAO, motor behavioral deficit scores were significantly higher in mice subjected to MCAO.Glycyrrhizin pre-treatment exhibited no significant reduction in neuronal deficit scores versus the MCAO control group (Figure 2(A)). Regions of ischemic infarction were confirmed by TTC staining, and MCAO was found to induce severe damage in ipsilateral hemispheres. However, mice pre-treated with glycyrrhizin $(10,30$, or $100 \mathrm{mg} / \mathrm{kg}$ ) had significantly smaller infarct lesions than MCAO controls (Figure 2(B)).

(A)

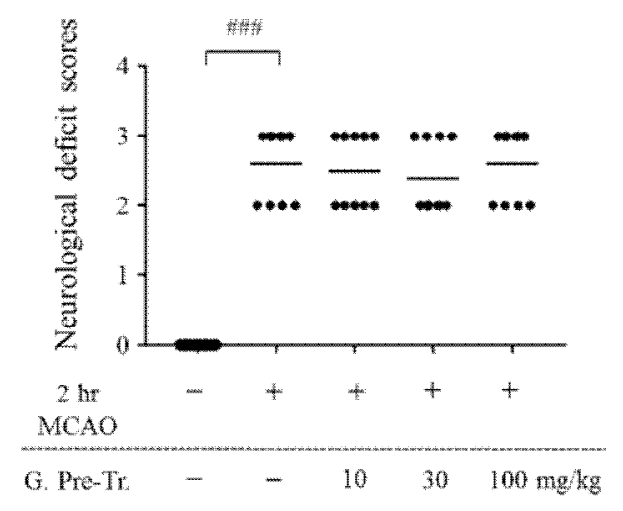

(B)

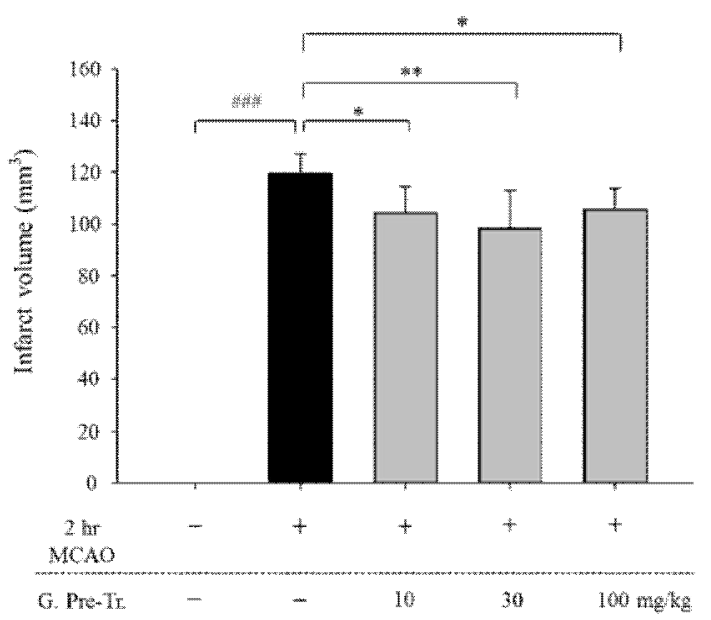

Figure 2: Effects of glycyrrhizinon neurological deficit scores and infarct volumes.Glycyrrhizin pretreatment did not improve neuronal deficit scores (A), but significantly decreasedinfarct volumes at $24 \mathrm{~h}$ post-MCAO (B). Results are presented as means \pm SDs. ${ }^{\# \#} \mathrm{p}<0.001$ vs. Sham controls, ${ }^{*} \mathrm{p}<0.05$, ${ }^{* *} \mathrm{p}<0.01$ vs. MCAO controls; $n=8$ in (A), $n=6$ in (B).

\section{Effects of glycyrrhizin on the expressions of apoptosis related proteins}

In ipsilateral hemispheres of MCAO subjected brains, western blot analysis at 24-post MCAO revealed Bcl-xLlevels were significantly lower than in sham controls (Figure 3). However, mice in the 10, 30 , and $100 \mathrm{mg} / \mathrm{kg}$ groups hadsignificant higher Bcl-xLlevels than mice in the sham control group (Figure 3). Glycyrrhizin pre-treatment down-regulated caspase-3 and caspase-9 in ischemic brains, but caspase- 8 levels were similar in all five study groups (Figure 4).

Western blot analysis at 24h-post MCAO revealed Bcl-2 expression was significantly lower in all MCAO groups than in sham controls (Figure 5). 
(A)
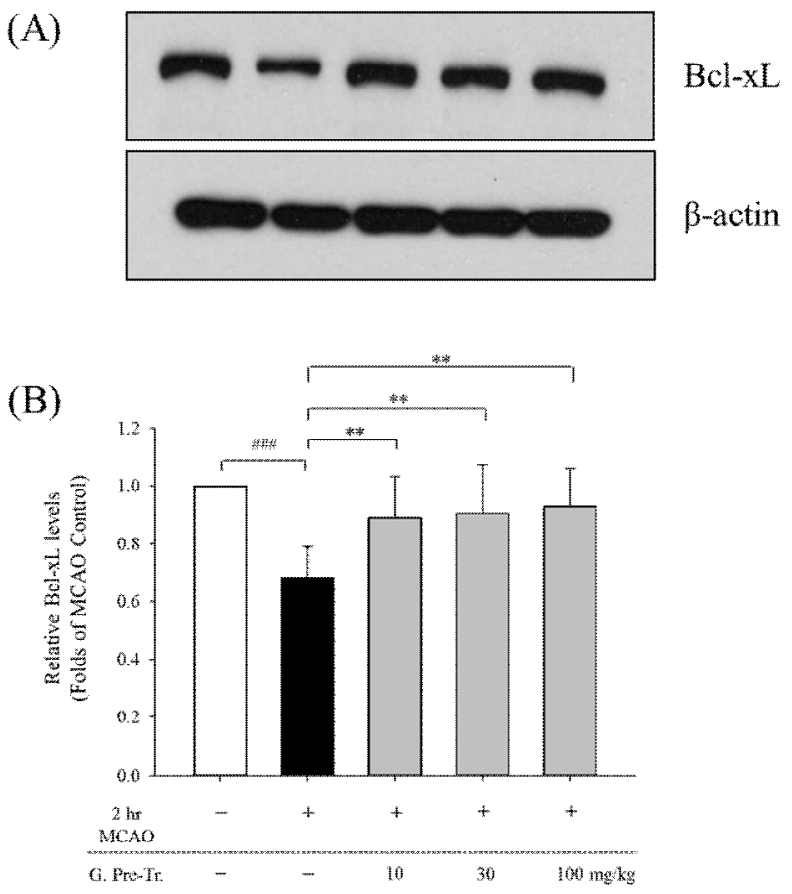

Figure 3: Effects of glycyrrhizin on Bcl-xLlevelsin the brains of MCAO-induced mice. Western blotting showedglycyrrhizin blocked the down-regulation of Bcl-xLby MCAO. Results are presented as means \pm SDs. ${ }^{\#} \mathrm{p}<0.001$ vs. Sham controls, ${ }^{* *} \mathrm{p}<0.01$ vs. MCAO controls; $\mathrm{n}=5$.

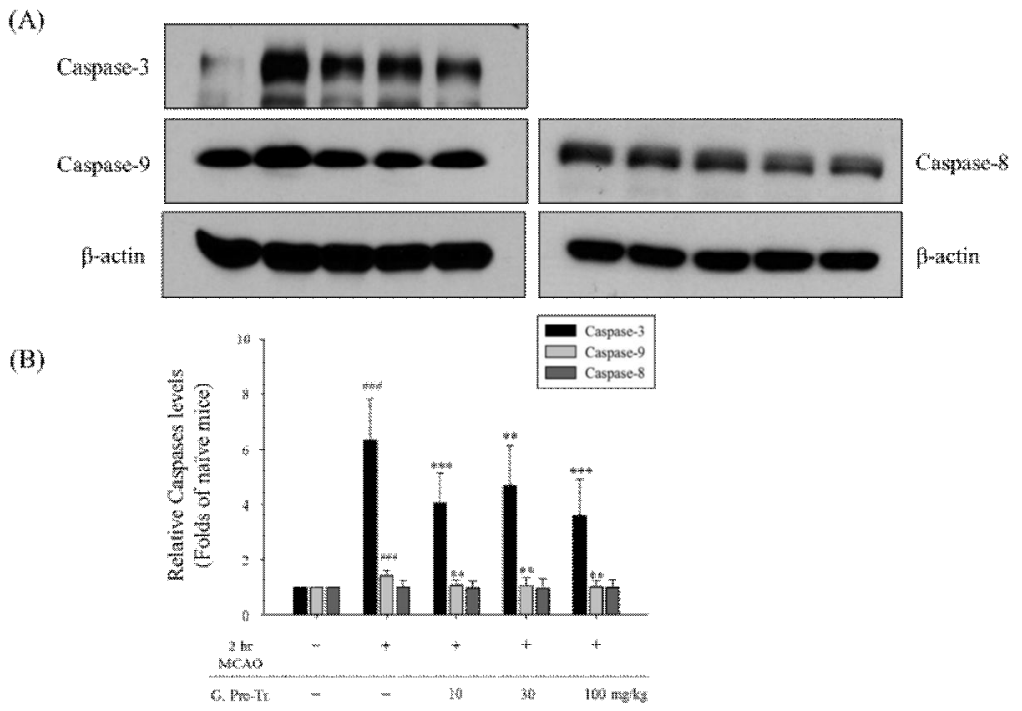

Figure 4: Effects of glycyrrhizin on brain caspase levels. Caspase-3, -8, and -9 were assessed by Western blotting.Glycyrrhizinwas found to block the MCAO-induced up-regulations of caspase-3 and -9. Results are presented as means \pm SDs. ${ }^{\# \# \#} \mathrm{p}<0.001$ vs. Sham controls, $* * \mathrm{p}<0.01, * * * \mathrm{p}<0.001$ vs. MCAO controls; $\mathrm{n}=5$. 
(A)

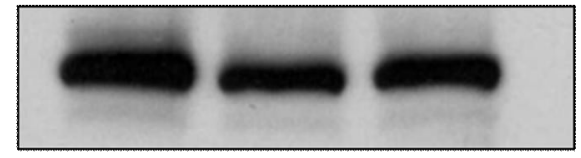

$\mathrm{Bcl}-2$
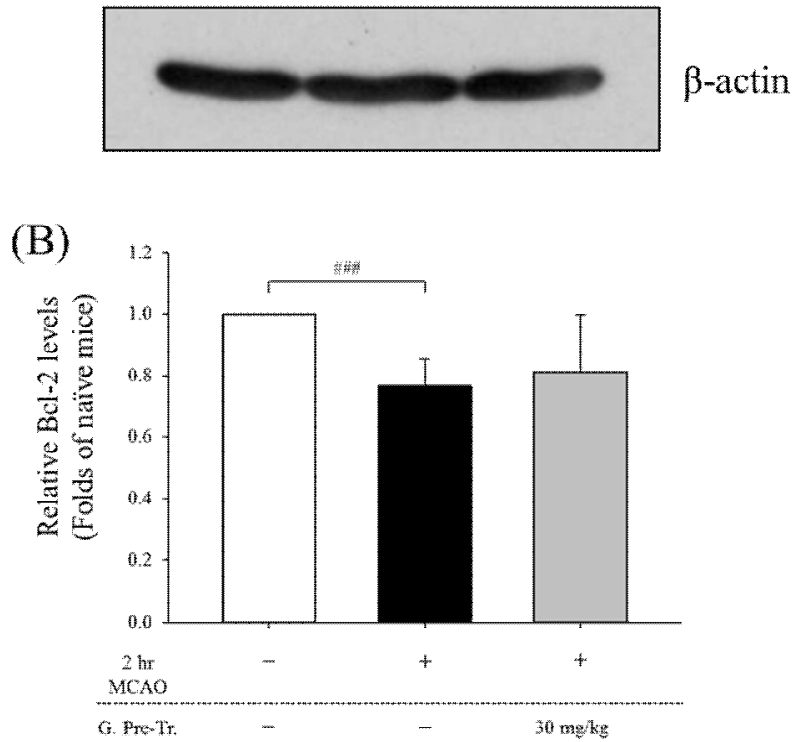

Figure 5: Effects of glycyrrhizin on brain Bcl-2 levels. Western blotting showed Bcl-2 levels were suppressed by MCAO and that glycyrrhizinhad no effect on this suppression. Results are presented as means \pm SDs. ${ }^{\# \# \#}$ p 0.001 vs. Sham controls; $n=5$.

\section{Discussion and Conclusion}

Ischemia-induced brain damage, also called "stroke" or "brain attack", is associated with oxidative stress and neuro-inflammation, in turn, cause diverse motor and speechdisorders (Chamorro et al., 2016; Roth andLiesz, 2016). A stroke can occur at any time, and in the USA, nearly 800,000 people experience a new or recurrent stroke annually.Stroke is the leading cause of adult disability and the fifth-most leading cause of death(Bejotet al., 2016; Hachinski andAzarpazhooh, 2016; Poisson et al., 2014). The severities of motor and speech disordersdepend on the extent of brain damage(Barlow, 2016), and thus, anti-apoptotic neuroprotective therapeutic agents offer an attractive developmental strategy.

In a preliminary study, we observed oral glycyrrhizin reducedbrain infarct volumes in MCAOtreated mice, and in the present study, single doses of glycyrrhizinof from 10 to $100 \mathrm{mg} / \mathrm{kg}$ produced similar results (Figures 1 and 2). In order to identify beneficial effects of glycyrrhizin, we assessed the levels of several apoptosis-related proteins and their activities in ipsilateral brains.

Caspases are classified based on the roles they play during apoptosis.Caspases- 8 and -9 are called "initiator caspases", because they activate executioner caspases (caspases $-3,-6$, and -7), which induce the destruction ofkey structural proteins. Caspase-3 is responsible for DNA fragmentation and plays a critical role in apoptosis (Mcllwain et al., 2015; Sun et al., 2015). Recently, several researchers have suggested ischemia/reperfusion-induced brain injury might be ameliorated by inhibiting caspase-3 signaling (Wen $e t$ al., 2016; Yang et al., 2015a), and in the present study, glycyrrhizinwas found to inhibit increase in caspase-3 and -9 levelscaused by MCAO-induced brain damage (Figure 4).

The Bcl-2 and caspase families play act as regulators of the apoptotic pathway, and interestingly, members of this family act as positive or negative regulators. Bcl-xL is an anti-apoptotic member of the Bcl2 family (Dragovich et al., 1998; Wu and Tang, 2016), and in the present study, its levels, and those of Bcl2 , 
were significantly lower in damaged brains.Furthermore, glycyrrhizinpretreatment effectively inhibited reduced this MCAO-induced Bcl-xLdown-regulation (Figure 3, Figure 5).

Our results suggest that glycyrrhizinpretreatment decreasedinfarct volumesin the brains of MCAOtreated miceby regulating apoptosis-related protein signaling (Figure 6). Accordingly, our findings indicate that glycyrrhizinis a promising candidate for the prevention or treatment of ischemia-induced brain damage.



Figure 6: Schematic of the anti-apoptotic action effect of glycyrrhizin in the MCAO mouse model. Red arrows indicate predicted effects of glycyrrhizin on ischemia/reperfusion induced brain damage.

\section{References}

1. Barlow, S. J. (2016). Identifying the brain regions associated with acute spasticity in patients diagnosed with an ischemic stroke. Somatosensory \& Motor Research1-8.

2. Bejot, Y., Delpont, B., andGiroud, M. (2016). Rising stroke incidence in young adults: More epidemiological evidence, more questions to be answered. Journal of the American Heart Association 5(5): 10.1161/JAHA.116.003661.

3. Chamorro, A., Dirnagl, U., Urra, X., andPlanas, A. M. (2016). Neuroprotection in acute stroke: Targeting excitotoxicity, oxidative and nitrosative stress, and inflammation. Lancet,Neurology 15(8):869-881.

4. Dragovich, T., Rudin, C. M., and Thompson, C. B. (1998). Signal transduction pathways that regulate cell survival and cell death. Oncogene 17(25):3207-3213.

5. Faraji, J., Lehmann, H., Metz, G. A., and Sutherland, R. J. (2009). Stress and corticosterone enhance cognitive recovery from hippocampal stroke in rats. Neuroscience Letters, 462(3):248-252.

6. Feigin, V. L., Anderson, N., Rinkel, G. J., Algra, A., van Gijn, J., and Bennett, D. A. (2005). Corticosteroids for aneurysmal subarachnoid haemorrhage and primary intracerebral haemorrhage. The Cochrane Database of Systematic Reviews (3):CD004583.

7. Guo, N. (1991). Protective effect of glycyrrhizin in mice with systemic candida albicans infection and its mechanism.Zhongguo Yi XueKeXue Yuan Xue Bao.Acta AcademiaeMedicinaeSinicae, 13(5):380-383.

8. Hachinski, V., andAzarpazhooh, M. R. (2016). Stroke is a burdensome but preventable brain disorder. Lancet,Neurology DOI:http//dx.doi.org/10.1016/S1474-4422(16)30120-X.

9. Hadadha, M., Vakili, A., andBandegi, A. R. (2015). Effect of the inhibition of hydrogen sulfide synthesis on ischemic injury and oxidative stress biomarkers in a transient model of focal cerebral ischemia in rats. 
Journal of Stroke and Cerebrovascular Diseases: The Official Journal of National Stroke Association 24(12): 2676-2684.

10. Ji, S., Li, Z., Song, W., Wang, Y., Liang, W., Li, K., and Ye, M. (2016). Bioactive constituents of Glycyrrhiza uralensis (licorice): Discovery of the effective components of a traditional herbal medicine. Journal of Natural Products, 79(2):281-292.

11. Kim, S. W., Jin, Y., Shin, J. H., Kim, I. D., Lee, H. K., Park, S., and Lee, J. K. (2012). Glycyrrhizic acid affords robust neuroprotection in the postischemic brain via anti-inflammatory effect by inhibiting HMGB1 phosphorylation and secretion. Neurobiology of Disease 46(1):147-156.

12. Kim, S. W., Lim, C. M., Lee, H. K., and Lee, J. K. (2011). The use of stronger neo-minophagen C, a glycyrrhizin-containing preparation, in robust neuroprotection in the postischemic brain. Anatomy \& Cell Biology 44(4):304-313.

13. Kumagai, A., Yano, S., andOtomo, M. (1957). Study on the corticoid-like action of glycyrrhizin and the mechanism of its action.Endocrinologia Japonica 4(1):17-27.

14. Luo, L., Jin, Y., Kim, I. D., and Lee, J. K. (2013). Glycyrrhizin attenuates kainic acid-induced neuronal cell death in the mouse hippocampus. Experimental Neurobiology 22(2):107-115.

15. Mcllwain, D. R., Berger, T., andMak, T. W. (2015). Caspase functions in cell death and disease. Cold Spring Harbor Perspectives in Biology 7(4):10.1101/cshperspect.a026716.

16. Ota, M., Mikage, M., and Cai, S. Q. (2015). Herbological study on the medicinal effects of roasted licorice and honey-roasted licorice. YakushigakuZasshi 50(1):38-45.

17. Poisson, S. N., Glidden, D., Johnston, S. C., and Fullerton, H. J. (2014). Deaths from stroke in US young adults, 1989-2009. Neurology 83(23): 2110-2115.

18. Roth, S., andLiesz, A. (2016). Stroke research at the crossroads - where are we heading? Swiss Medical Weekly146:w14329.

19. Sandercock, P. A., and Soane, T. (2011). Corticosteroids for acute ischaemic stroke. The Cochrane Database of Systematic Reviews (9):CD000064.

20. Segal, R., Pisanty, S., Wormser, R., Azaz, E., andSela, M. N. (1985). Anticariogenic activity of licorice and glycyrrhizin I: Inhibition of in vitro plaque formation by streptococcus mutans. Journal of Pharmaceutical Sciences 74(1):79-81.

21. Sun, Y., Xu, Y., andGeng, L. (2015). Caspase-3 inhibitor prevents the apoptosis of brain tissue in rats with acute cerebral infarction. Experimental and Therapeutic Medicine 10(1):133-138.

22. Wen, X. R., Fu, Y. Y., Liu, H. Z., Wu, J., Shao, X. P., Zhang, X. B. \&Song, Y. J. (2016). Neuroprotection of sevoflurane against ischemia/reperfusion-induced brain injury through inhibiting JNK3/caspase-3 by enhancing Akt signaling pathway. Molecular Neurobiology 53(3):1661-1671.

23. Wu, Y., and Tang, L. (2016). Bcl-2 family proteins regulate apoptosis and epithelial to mesenchymal transition by calcium signals. Current Pharmaceutical Design 22(30):4700-4704.

24. Yang, P. S., Lin, P. Y., Chang, C. C., Yu, M. C., Yen, T. L., Lan, C. C., andYang, C. H. (2015a). Antrodiacamphorata potentiates neuroprotection against cerebral ischemia in rats via downregulation of iNOS/HO-1/bax and activated caspase-3 and inhibition of hydroxyl radical formation. Evidence-Based Complementary and Alternative Medicine Article 232789.

25. Yang, R., Wang, L. Q., Yuan, B. C., and Liu, Y. (2015b). The pharmacological activities of licorice. Planta Medica 81(18):1654-1669.

26. Zhan, C., and Yang, J. (2006). Protective effects of isoliquiritigenin in transient middle cerebral artery occlusion-induced focal cerebral ischemia in rats. Pharmacological Research 53(3): 303-309.

27. Zhao, P., Zhou, R., Zhu, X. Y., Hao, Y. J., Li, N., Wang, J., and Yu, J. Q. (2015). Matrine attenuates focal cerebral ischemic injury by improving antioxidant activity and inhibiting apoptosis in mice. International Journal of Molecular Medicine 36(3):633-644.

28. Zhu, Z., Tao, W., Li, J., Guo, S., Qian, D., Shang, E., andDuan, J. A. (2016). Rapid determination of flavonoids in licorice and comparison of three licorice species. Journal of Separation Science 39(3):473-482. 